Article

\title{
First-Order Peaks Determination for Direction-Finding High-Frequency Radar
}

\author{
Yeping Lai ${ }^{1}\left(\mathbb{D}\right.$, Yuhao Wang ${ }^{1, *}$ and Hao Zhou ${ }^{2} \mathbb{D}$ \\ 1 The School of Information Engineering, Nanchang University, Nanchang 330031, China; yeping.1@ncu.edu.cn \\ 2 The School of Electronic Information, Wuhan University, Wuhan 430072, China; zhou.h@whu.edu.cn \\ * Correspondence: wangyuhao@ncu.edu.cn
}

check for

updates

Citation: Lai, Y.; Wang, Y.; Zhou, H. First-Order Peaks Determination for Direction-Finding High-Frequency Radar. J. Mar. Sci. Eng. 2021, 9, 8. https://dx.doi.org/10.3390/ jmse 9010008

Received: 21 November 2020 Accepted: 18 December 2020 Published: 23 December 2020

Publisher's Note: MDPI stays neutral with regard to jurisdictional claims in published maps and institutional affiliations.

Copyright: () 2020 by the authors. Licensee MDPI, Basel, Switzerland. This article is an open access article distributed under the terms and conditions of the Creative Commons Attribution (CC BY) license (https: / / creativecommons.org/ licenses/by/4.0/).

\begin{abstract}
Direction-finding (DF) high-frequency radar (HFR) is preferred among the HFR family and is widely used around the world due to its compact structure. The correct determination of first-order peaks (FOPs) from Doppler spectra recorded by radar is a critical step toward attaining accurate mappings of surface currents. The commonly used FOPs determination method is generally sufficient for most situations. However, it needs six user-defined input parameters. These parameters result in complex procedures of optimizing the values of these six user-defined parameters. To simplify the FOPs determination for DF HFR, we propose an alternative method which only needs one user-defined parameter. To validate the reliability of the proposed method, we compare the FOPs determination results derived from the proposed method with those from the commonly used method on a data set covering a period of 256 days. The results indicate that the proposed method yields a similar FOPs determination result to the commonly used method. This proposed inputparameter-reduced method can greatly simplify the use of the HFR for users who are unprofessional in the HFR and promote the popularization and application of HFR.
\end{abstract}

Keywords: high-frequency radar; first-order peaks determination; SeaSonde method; first-order region boundary

\section{Introduction}

High-frequency radar (HFR), which works at a frequency of 3 to $30 \mathrm{MHz}$, is widely used to remotely sense oceanic surface state [1-4]. By combining Bragg coherent scattering and Doppler shift, HFR can provide the sea surface current field over a large area, with high spatial $[O(1 \mathrm{~km})]$ and temporal $[O(1 \mathrm{~h})]$ resolutions [5-8]. At present, HFR systems are roughly divided into two groups according to their receiving antennas, i.e., beam forming radar equipped with a phased array antenna (e.g., Wellen Radar (WERA) [9]) and directionfinding (DF) radar adopting a compact monopole-cross-loop antenna (e.g., Coastal Ocean Dynamics Application Radar (CODAR) SeaSonde $[10,11])$. Due to the compact physical structure, the DF HFR is more widely deployed around the world [12].

In DF HFR systems, the accurate separation of the first-order peaks (FOPs) is a critical step for attaining accurate sea surface currents [13].Because inaccurate FOPs separation will result in either omission of some accurate radial current observations or spurious radial current observations. To automatically separate the FOPs from a range-dependent Doppler spectrum for DF HFR, the logarithmic difference spectrum method was developed by Lipa and Barrick in 1983 [11]. At present, this logarithmic difference spectrum method is completely replaced by an empirical method. This empirical method was first presented in a SeaSonde technical manual in 2002 [14]. Now, it is routinely used in the SeaSonde system. In our work, we refer to this empirical method as the SeaSonde method and sometimes the commonly used method for convenience. The SeaSonde method relies on six user-defined parameters. (In the SeaSonde Radial Suite [15], they are named noisefact, nsm, nsec, flown, flim, and currmax. The detailed descriptions of these parameters are 
presented in [15] also in Appendix A. In general, this methodology is sufficiently accurate for FOPs identification in most cases. However, the process of optimizing the values of the six user-defined parameters is complicated, because some parameters have only a minor effect (i.e., $n s m$ ) and others (noisefact, flim) have overlapping effects [16]. Recently, to reduce the complexity of FOPs determination for broad-beam DF HFR, Kirincich [16] proposed an image-processing-based (IPB) FOPs determination method, which needs three userdefined parameters. Therefore, this IPB method reduces input parameters and simplifies the parameter tuning process. To assess the performance of this IPB method, Kirincich [16] compared this IPB method with the SeaSonde method by visually inspecting the FOPs determination results and evaluating the accuracy of radar-derived radial velocities. The radial velocities evaluation is convincing, whereas the visual inspection is not objective enough as well as time and labor consuming. Because in some cases the spectra are very complex, which results in visually determining the true FOPs is questionable even for experts.

In this paper, we propose an alternative FOPs determination method and explore more objective and more efficient methods than the visual inspection method for assessing the performance of FOPs determination methods. The proposed FOPs determination method uses the averaged amplitude of a portion of second-order peaks as an amplitude threshold to determine FOPs in the case of second-order echoes existing and uses a constant signal-to-noise ratio (SNR) to separate FOPs in the case of second-order echoes absent. In this work, we refer to this proposed method as the second-order spectra-based (SSB) FOPs determination method. The SSB method only needs to optimize one user-defined parameter, which is the maximum current velocity that may be encountered by the radar. A MATLAB-based software package that performs the SSB method is being made publicly available in https://github.com/260846137/SSB-FOPS.git to facilitate additional testing and further improvements. The performance of the SSB method is checked by comparing the FOPs determination results from the SSB method with those from the SeaSonde method. The method of assessing the performance of the SSB method is totally different from the visual inspection method used in [16]. In this study, we analyze the boundaries and the number of solutions of the determined FOPs. Apparently, these analyses are more objective than the visual inspection. Thus, the novelties of this work are to present an alternative FOPs separation method and provide a new approach for assessing the performance of a FOPs separation method.

The paper is organized as follows: the details on the proposed FOPs determination method (the SSB method) is presented in Section 2, followed by a statement of the data set and the testing results in Section 3; a discussion of the testing results of the SSB method is presented in Section 4; following the discussion, we present conclusions of this paper in Section 5 .

\section{The Proposed SSB Method}

Doppler spectra received by HFR have dominant FOPs due to the scattering from the Bragg ocean waves with wavelength exactly one half of the radar wavelength. Moreover, the FOPs are surrounded by second-order echoes, predominantly caused by the interaction of the radar wave with pairs of ocean waves. However, the second-order echoes are absent in some region (e.g., in a harbor), where there are no long waves. Moreover, Doppler spectra backscattered from far range cells without second-order returns are common. For the case of having second-order returns, the SSB method uses the appearance characteristics of radar-received Doppler spectra that contain nulls between first- and second-order spectra. Furthermore, second-order spectra are typically an order of magnitude below FOPs. Thus, the Doppler bins at the periphery of the first-order region (FOR) with amplitude close to the null-neighboring second-order spectral points can be used as the FOR boundaries. Unfortunately, the null-neighboring second-order spectrum region is difficult to extract, because the profile of second-order spectra is not constant due to the variation of sea state. Nevertheless, as long as the second-order returns are 
present, there are always singular peaks within the second-order region, and they have a Doppler frequency of invariably around $\sqrt{2}$ times Bragg frequency [17-19]. Thus, we can easily locate a portion of second-order peaks in a conservative way, i.e., locating singular peaks. In addition, the amplitude of the second-order region is range-dependent, which is just like the FOPs. As a part of second-order spectra, the singular peaks also have range-dependent amplitudes. In this study, we exactly use the averaged amplitudes of the range-dependent singular peaks as a threshold to extract FOR for the case of having secondorder returns, so the threshold can be adaptively adjusted according to the amplitude of the range-dependent first- and second-order peaks. For the case without second-order returns, we use a constant SNR to extract the FOR. In this study, the SNR is defined as the power of spectral point to noise level. To be able to handle these two cases, the SSB method automatically diagnoses whether a spectrum belongs to the case of having second-order returns or without second-order returns.

To clearly show the ability of the proposed method in determining FOPs, we intend to check the difference of the FOPs determination results between the SeaSonde method and the proposed method. The description of the SeaSonde method as well as its settings are presented in Appendix A. Here, we present the detailed calculations of the proposed SSB determination method. We first calculate the noise level, $N_{T}$, which is calculated with the same method as the SeaSonde method (i.e., step 1 for the SeaSonde method in Appendix A). The Doppler bins with power less than $N_{T}$ plus $8 \mathrm{~dB}$ (this SNR theoretically results in a bearing error less than two degrees for a monopole-cossed loop antenna [20]) will be eliminated. Then, the maximum current velocity is used to determine the proposed Doppler bins, which include all the true FOPs and often include some second-order peaks. The set for indexing these proposed Doppler bins can be expressed as

$$
A=\left\{i \in N_{s} \mid \frac{\lambda}{2}\left\|f_{i}-f_{b}\right\| \leq v_{\max }\right\},
$$

where $N_{s}=\{1,2, \ldots, N\}$ is the index of the Doppler bins, sorted by Doppler frequency, backscattered from one range cell, and $N$ is the count of the Doppler bins; $\lambda$ is the radar wavelength; $f_{i}$ denotes the frequency of the ith Doppler bin; $f_{b}$ is the Bragg frequency; and $v_{\max }$ is the maximum current velocity threshold, which is determined by historical observation data. For an observation experiment, we can determine the $v_{\text {max }}$ by manually inspecting the collected Doppler spectra. In this study, we randomly inspected some spectra within our data set (see Section 3.1) and found that the Doppler spectra always have a maximum radial current velocity being less than $1 \mathrm{~m} / \mathrm{s}$. To ensure that all the true FOPs do not be missed, $v_{\max }$ is set as $1.5 \mathrm{~m} / \mathrm{s}$ for this study. For a routinely operation HFR site, the $v_{\max }$ can be determined by finding the historical maximum current velocity recorded by any current measurement instrument or consulting the users, who intend to use the HFR-output current mapping products. In the next step, we search for the Doppler bin, which has a maximum power within $A$. Then, a window, centered on $\sqrt{2}$ times the frequency of the determined maximum power bin, with a length of $L$ Doppler bins, is used to extract a portion of second-order peaks. An offset, $d$, is defined as the absolute value of the edge of the window to the center. Thus, we have

$$
L=2 d+1
$$

where both $L$ and $d$ are positive integers. The amplitude threshold is calculated as the averaged amplitude of these $L$ Doppler bins to eliminate the second-order peaks included in set $A$. Thus, the amplitude threshold, $T$, can be written as

$$
T=\frac{1}{L} \sum_{j=-d}^{d} p\left(I_{B}+j\right),
$$


where $I_{B}$ is the index of the spectral point that has a frequency being equal to $\sqrt{2}$ times the frequency of the maximal power bin and $p(\cdot)$ denotes the signal amplitude of the Doppler bin.In our work, we empirically set $d=3$. One reason is that the singular peaks are broadened due to sea surface currents, which has been theoretically proved [18] and experimentally testified $[21,22]$. Another reason is that the velocity resolution for a sea surface current observation HF radar always ranges from $2 \mathrm{~cm} / \mathrm{s}$ to $6 \mathrm{~cm} / \mathrm{s}$, so an offset of 3 corresponding to a velocity of $6 \mathrm{~cm} / \mathrm{s}$ to $18 \mathrm{~cm} / \mathrm{s}$, which are hardly greater than the maximum current velocity for a Doppler spectrum. Thus, the setting of $d=3$ (or $L=7$ ) can ensure the selected portion of second-order peaks are singular peaks and can eliminate the fluctuation of $T$ resulting from noise. In the following step, we calculate the amplitude (power) threshold. The greater one for $T$ and noise level plus 8 (i.e., $N_{T}+8$ ) is adopted as the threshold. Then, we search for the Doppler bins which are first less than this threshold, in the two sides around the Doppler bin, which has a maximum power within $A$. The boundaries can be expressed as

$$
\begin{aligned}
& l_{+}=I\left[p(i)<\max \left(T, N_{T}+8\right)\right], \text { with } \operatorname{minimum}\left(i-I_{p \max }\right) ; i \in A, \\
& l_{-}=I\left[p(i)<\max \left(T, N_{T}+8\right)\right], \text { with } \operatorname{minimum}\left(I_{p \max }-i\right) ; i \in A,
\end{aligned}
$$

where $l_{+}$and $l_{-}$are the right- and left-hand boundary for FOR, respectively; $I$ [expression] denotes the index of a Doppler bin that the corresponding power satisfies the expression; $I_{p \max }$ denotes the index of the Doppler bin with maximum power within set $A$; $\max \left(T, N_{T}+8\right)$ represents outputting the greater one for $T$ and $N_{T}+8$. In fact, the manipulation of adopting the greater one between $T$ and $N_{T}+8$ as the threshold is just the diagnosis, which diagnoses whether there is noteworthy second-order returns within the Doppler spectrum or not.

To easier understand the method described above, Figure 1 summarizes the process of the SSB method. The step 1 (calculating noise level) is applied to a whole Doppler spectrum, whereas the following four steps (step 2 to step 5) are applied separately for the positive and negative halves of a Doppler spectrum. In addition, two examples are provided in Figure 2 for illustrating the proposed FOPs determination method. The example in Figure 2a is a positive half of a Doppler spectrum collected at the range cell being $25 \mathrm{~km}$ off HFR. The power of the second-order spectra has a relatively high level. The averaged power of the selected portion of the second-order peaks $(T)$ is greater than $N_{T}$ plus 8, so the power threshold is equal to $T$. The example in Figure $2 \mathrm{~b}$ is a negative half of a Doppler spectrum for $50 \mathrm{~km}$ off HF radar. In this spectrum, there is no second-order sea echo, because the spectrum is collected at a too far range cell. Thus, the selected portion of the second-order spectra only contains noise, and the averaged power of the selected portion of the second-order peaks $(T)$ is close to the noise level $\left(N_{T}\right)$. In this case, the power threshold for determining FOPs is noise plus $8 \mathrm{~dB}$. The spectral points over the red shadowed sandwiched between the two black dots are the determined first-order Doppler bins. These Doppler bins will be used to map radial current field. 


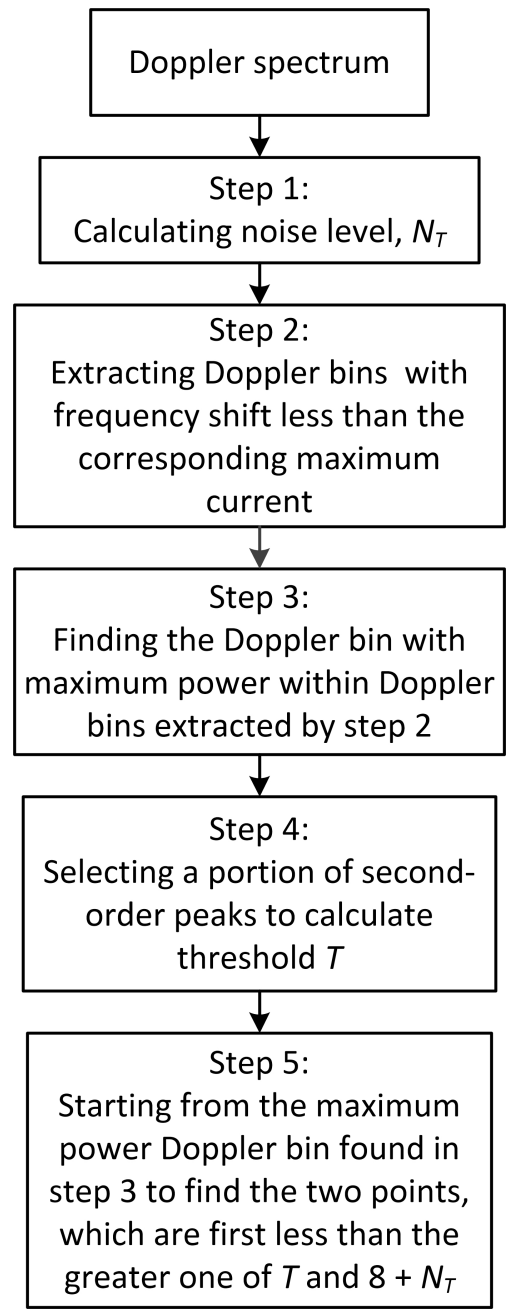

Figure 1. The processing flow of the second-order spectra-based (SSB) method.

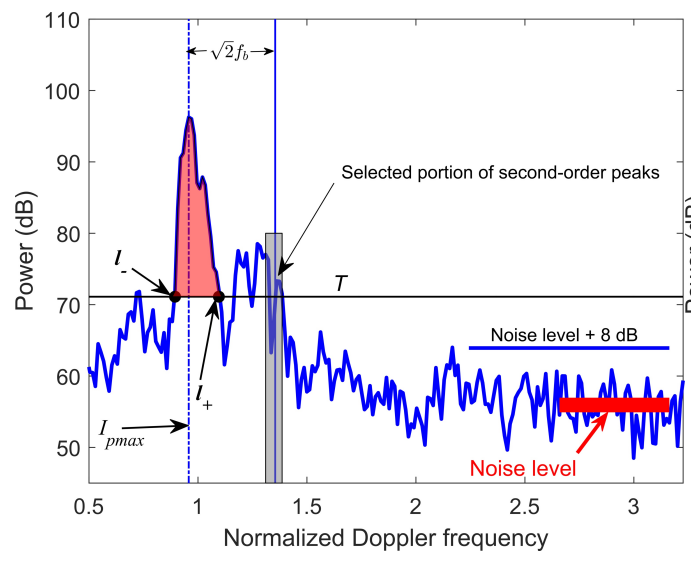

(a)

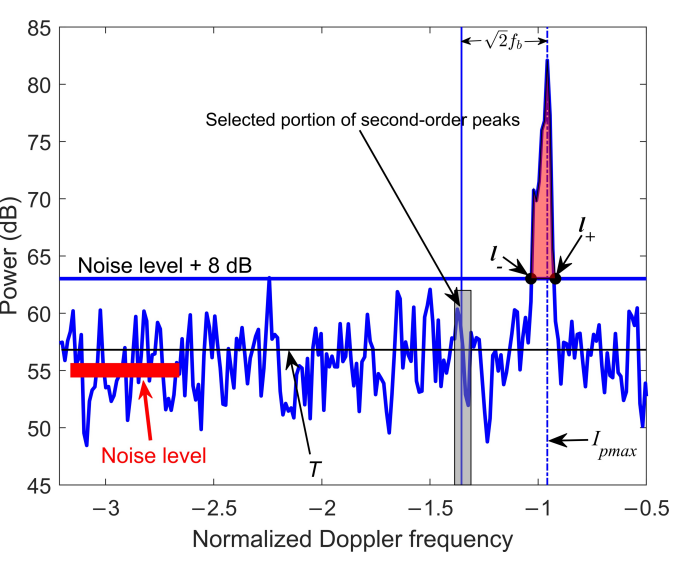

(b)

Figure 2. Demonstrates the first-order peaks determination for the second-order spectra-based (SSB) method. (a) a positive half Doppler spectrum case with prominent second-order spectra; (b) a negative half Doppler spectrum case without second-order spectrum. Apparently, in (b), the selected portion of second-order spectral points only contain noise, and without any second-order echo. $T$ is the mean power of the selected portion of second-order spectral points, which are indicated by a light gray shadowing rectangle centering on the blue vertical line. The spectral points within the two black solid dots are the determined FOPs. 


\section{Testing the SSB Method}

\subsection{Test Data Set}

From 11 January to 23 September 2019, a field observation experiment for measuring sea surface currents was conducted in Hainan Province, China. In this 256-day experiment, 41,111 range-Doppler spectra were collected. Thus, these vast amounts of Doppler spectra and the long-term observation time give a good chance to sufficiently validate the FOPs determination ability for the SSB method. The geographical map for the experiment is shown in Figure 3. The HFR system used in this experiment is a compact DF radar, named Ocean State Monitoring and Analyzing Radar, type S (OSMAR-S), which is developed by Wuhan University, China and commercially manufactured by Hainan Ruihai Ocean Technology Co. Ltd. [23]. This HFR system uses a compact antenna consisting of one monopole and two orthogonal crossed loops to receiving the sea echoes, just like the CODAR SeaSonde system [24,25]. During the experiment, the deployed HFR system transmitted at $16 \mathrm{MHz}$ with a bandwidth of $60 \mathrm{kHz}$. The corresponding range resolution was $2.5 \mathrm{~km}$. The designed routine detection range was $100 \mathrm{~km}$.

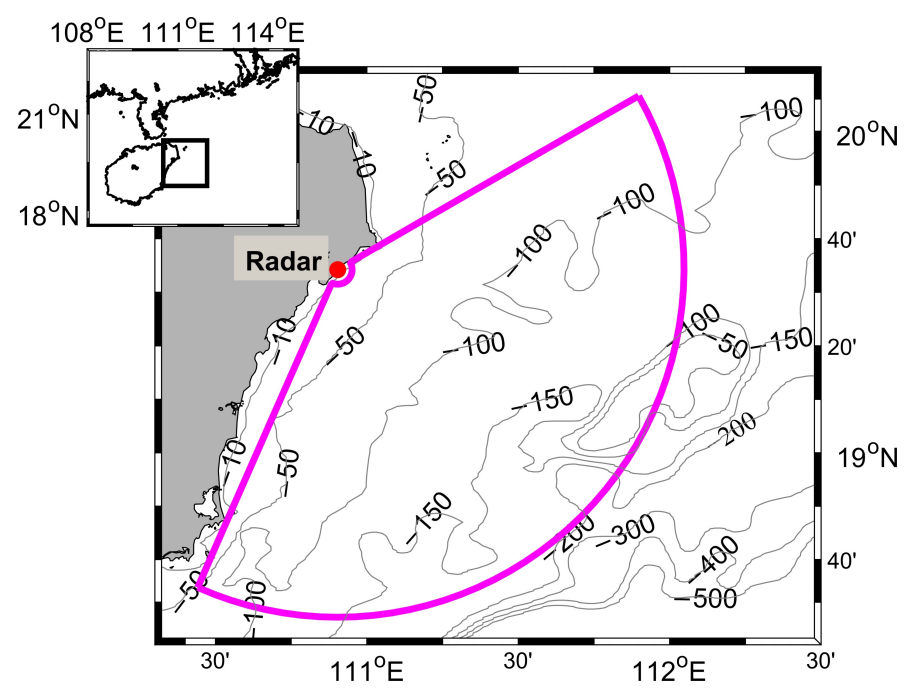

Figure 3. The geographical map for the field experiment. The thin gray lines denote isobaths. The red bullet indicates the location of our radar. The fanwise area enclosed by a thick pink line denotes the nominal observation area of the high-frequency radar (HFR) system.

\subsection{Results}

The performance of the SeaSonde method is recognized. To validate the performance of the SSB method, we check the differences of the FOPs determination results between the SSB method and the SeaSonde method. Here, we first compare the number of first-order Doppler bins determined by these two methods for each range-Doppler spectrum and then statistically analyze the radar-derived maximal and minimal radial currents. Finally, we analyze the performance of the SSB method on edge cases.

The number of the determined FOPs for each range-Doppler spectrum is calculated as the sum of the determined first-order spectral points for the Doppler spectrum collected from the 3rd to 40th range cell. Although the radar-derived maximal and minimal radial currents are corresponding to the right and left boundaries of determined FOR. Therefore, we calculate the radial velocity of the determined FOPs based on the difference between their Doppler frequencies and the Bragg frequency. Consequently, the left boundary and the right boundary of the determined FOPs are the minimal and maximal velocities, respectively. It should be noted that the minimal and maximal velocities are calculated for a whole Doppler spectrum, not calculated separately for positive and negative Doppler halves of a Doppler spectrum. Furthermore, the minimal velocities corresponding to the left boundary often represents the maximal radial current velocity flowing away from the radar (or the maximal negative radial current), whereas the maximal velocity corresponding to 
the right boundary often represents the maximal radial current velocity flowing towards the radar (or the maximal positive radial current).

Figure 4 shows the scatter plot of the number of the FOPs derived from the SeaSonde method $\left(N_{\text {cum }}\right)$ for each range-Doppler spectrum versus that from the SSB method $\left(N_{\text {pro }}\right)$. This figure indicates that the SSB method produces a similar result in the SeaSonde method with respect to the number of the determined FOPs. However, the fitted line does not exactly overlap with the baseline. Comparing the fitted line with the baseline in Figure 4, we can easily see that the fitted line almost overlaps with the baseline for $N_{\text {cum }}$ being less than 500 and above the baseline with a growing gap for $N_{\text {cum }}$ being greater than 500 . This phenomenon indicates that the SSB method produces almost the same number of FOPs as the SeaSonde method for Doppler spectra with narrow FOR and more FOPs than the SeaSonde method for Doppler spectra with relatively broad FOR. These differences between these two methods result from the fact that a broader FOR is more likely to contain local minima within the tails of the FOR. These local minima tend to be misidentified as nulls for the SeaSonde method. Figure 5a shows the histograms of the maximal and minimal radial current velocities. The distribution of the maximal and minimal radial current velocities derived from the SSB method are similar to those derived from the SeaSonde method. Figure $5 b$ shows the histograms of the difference between the maximal radial current velocities derived from the SeaSonde method and those derived from the SSB method. The difference between the maximal radial current velocities is principally distributed within $-\Delta f_{D}$ to $\Delta f_{D}$. Here, the number of the samples with $\left|V_{m t c}-V_{m t p}\right| \leq$ $\Delta f_{D}$ and $\left|V_{\text {map }}-V_{\text {mac }}\right| \leq \Delta f_{D}$ respectively account for $79.65 \%$ and $80.79 \%$ of the total number of the samples. These results suggest that the boundaries of the FOR derived from the SSB method are similar to those extracted by the SeaSonde method. Thus, these results about the number of determined FOPs and the boundaries provide solid evidence for that the SSB method has a comparable ability with respect to the SeaSonde method in determining FOPs.

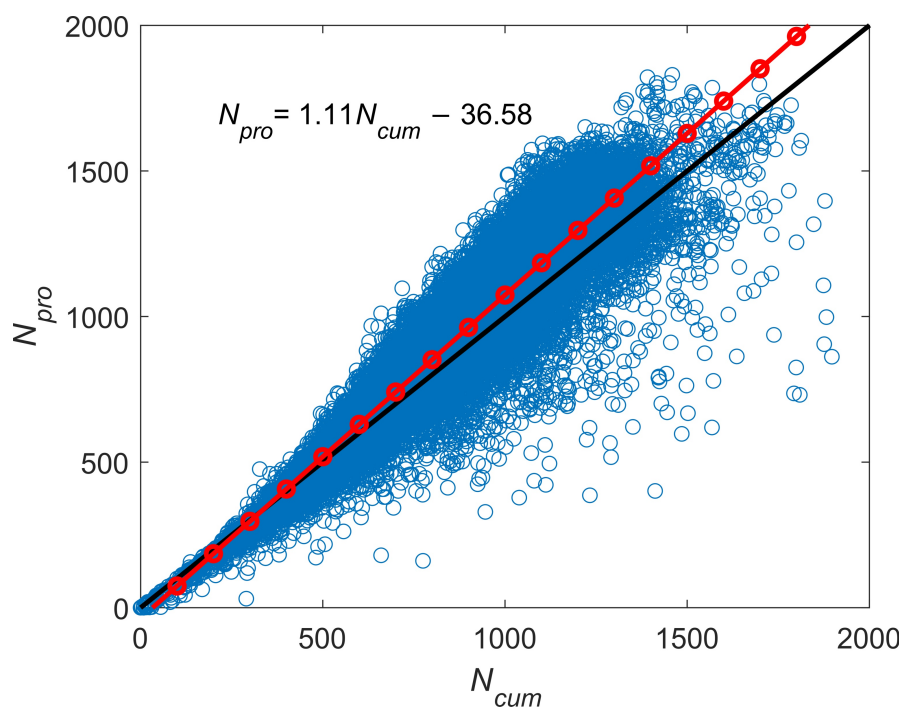

Figure 4. Scatter plot of the number of the first-order Doppler bins determined by the SeaSonde method $\left(N_{\text {cum }}\right)$ versus the number of the first-order Doppler bins determined by the second-order spectra-based method $\left(N_{\text {pro }}\right)$ for each range-Doppler spectrum. The red line marked with circle is the fitted line, and the black line is a baseline. 


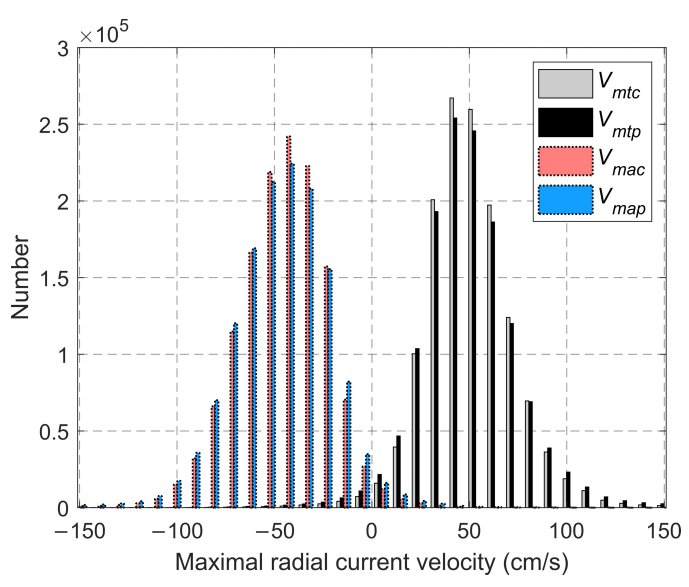

(a)

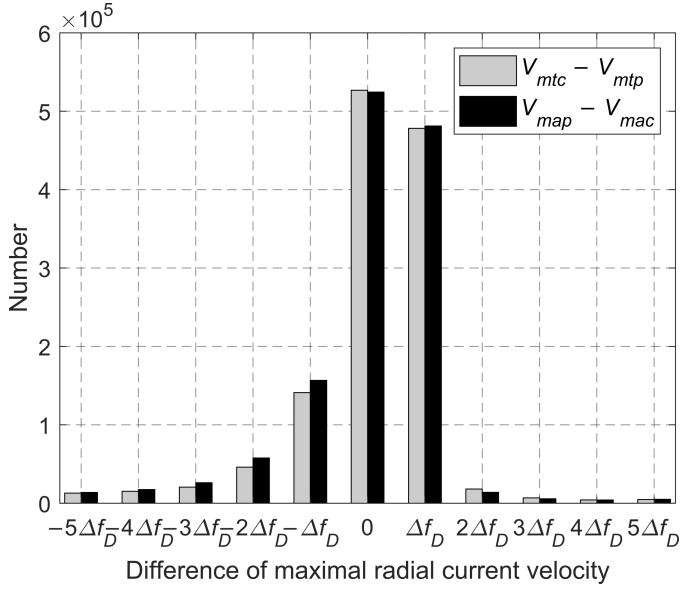

(b)

Figure 5. Histogram of (a) maximal and minimal radial current velocities for all range-dependent Doppler spectra and (b) the difference between the maximal (and minimal) radial current velocity derived from the SeaSonde method and that derived from the proposed method. $V_{m a c}$ and $V_{m t c}$ are the minimal and maximal radial currents extracted by the SeaSonde method, respectively. $V_{\text {map }}$ and $V_{m t p}$ are the minimal and maximal radial currents extracted by the proposed method, respectively. $\Delta f_{D}$ is the velocity resolution of our radar, which is $4.84 \mathrm{~cm} / \mathrm{s}$. Moreover, a small number of samples, for which the difference of the maximal (and minimal) radial current velocity is greater than $5 \Delta f_{D}$, is not shown in (b).

The above-mentioned statistical results suggest that the SSB method has a comparable ability with respect to the SeaSonde method. However, these statistical results do not include the comparison of the SSB method with the SeaSonde method in the edge cases, in which bifurcated peaks exist within FOR and FOPs overlap with second-order region, because the number of the spectral samples for the edge cases in our data set is very small. Here, we analyze the performance of the SSB method for the edge cases.

The bifurcated FOR, in which the FOR is divided into two or more groups by prominent local null, originates from the power fluctuation of the FOPs. Figure 6 shows a bifurcated FOR case with respect to the FOPs determination. In this figure, the FOR contains a significant null, which leads the FOR to a bifurcated profile. In addition, there is no second-order region for the displayed positive Doppler spectra. Thus, for the SSB method, the noise level plus $8 \mathrm{~dB}$ is the power threshold (just as Figure 2b). Starting from the maximum power Doppler bin, we can find the FOR boundary, where the power is first equal to or less than the power threshold. As Figure 6a shows, the determined FOPs (which is the region between the two red solid dots) are acceptable. For the SeaSonde method, the details of this method in determining FOPs for the bifurcated FOR case are illustrated in Figure $6 \mathrm{~b}$. The SeaSonde method handles the smoothed spectra. Starting from the two spectral points with the power of maximum power (MAXP) divided by fdown (MAXP/fdown), the SeaSonde method finds the two nulls indicated by the vertical lines. Then, the determined FOPs can be obtained based on the power threshold. Thus, the FOPs between the two red solid dots are selected by the SeaSonde method. However, nearly half true FOPs are falsely missed. For this spectrum, the SSB method obviously yields a more reliable FOPs determination result than the SeaSonde method. This unsatisfactory result for the SeaSonde method results from the bifurcated first-order, which has a large difference between the maximum power and the power of the spectral points in the local null. In fact, the SeaSonde method expectedly yields an unsatisfactory FOPs determination result as long as the power of the spectral point within the null is less than MAXP/fdown. Definitely, the SSB method also will yield an unsatisfactory result, if the power of the spectral point within the local null is less than noise level plus $8 \mathrm{~dB}$. Nevertheless, it is more common for the spectral points within local null with power less than MAXP/fdown than it less than the 
noise level plus $8 \mathrm{~dB}$, because MAXP/fdown almost definitely greater than the noise level plus $8 \mathrm{~dB}$. Thus, the SeaSonde method is more sensitive to local null within FOR than the SSB method. In other words, the SeaSonde method more likely yields unsatisfactory FOPs determination result with some true FOPs omitted than the SSB method for a bifurcated FOR spectrum.

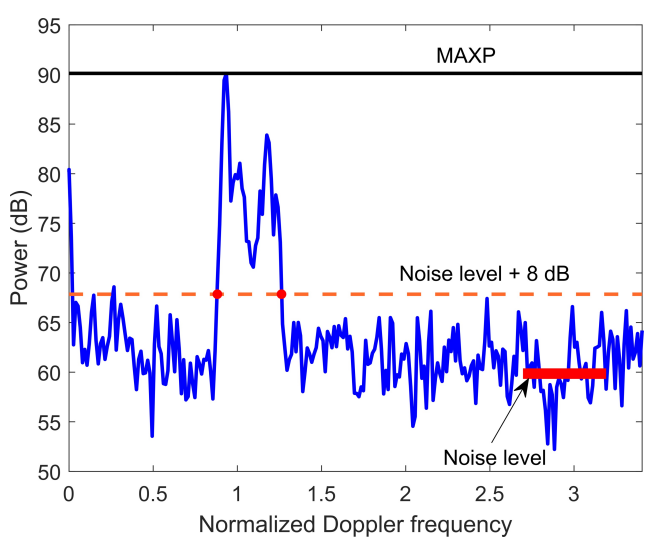

(a)

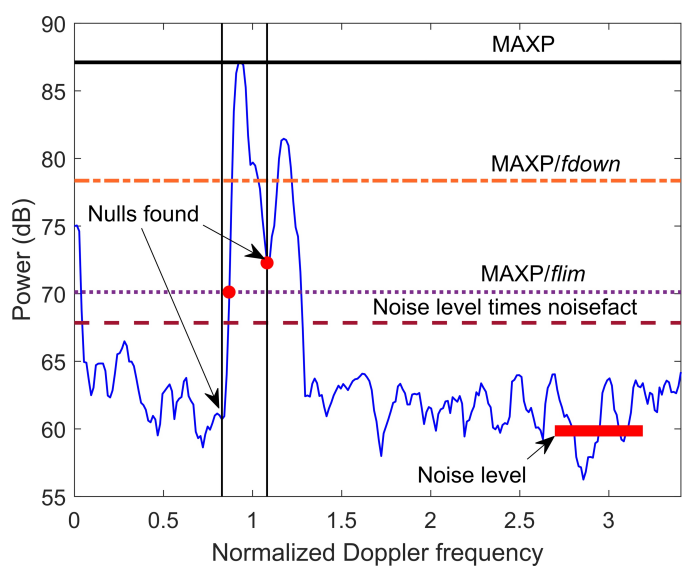

(b)

Figure 6. First-order peaks determination for bifurcated first-order region case using (a) the proposed method and (b) the SeaSonde method. The spectral points between the two red solid dots are the determined first-order region. In addition, MAXP is the maximum power. The detailed setting of the parameters flown and flim are stated in Appendix A.

FOPs overlapping with second-order region occurs, when the ground-truth radial current within radar observation area is very strong or the long oceanic wave (swell) components are strong. Also, if FOPs overlap with second-order region, the null between the FOR and the second-order region will not exist and the FOR will suddenly drop to the second-order region. Figure 7 shows an example of FOPs determination for the edge case of FOPs overlapping with second-order region. From Figure 7a we can clearly see that the FOR suddenly drops to the second-order region and there is no prominent null between the FOR and the second-order region. The FOPs determination results derived from the SSB method and the SeaSonde method are respectively illustrated in Figure 7a,b. The spectral points between the red solid square and the red solid dot are the determined FOPs, whereas the spectral points between the red solid square and the black solid square are the visually determined FOPs. Specifically, the spectral points between the black solid square and the red solid dot are second-order sea echoes and misidentified as FOPs. In fact, for the SSB method, when the null between the first- and second-order region is absent, the power of the spectral points in the extreme edge of the FOR will no longer be less than the calculated power threshold (e.g., Figure 7a). Thus, some second-order spectral points between FOR and the selected portion of second-order peaks may be misidentified as FOPs. On the other hand, because of the moving average manipulation for the Doppler spectrum, the null found by the SeaSonde method will also within in second-order region (e.g., Figure $7 \mathrm{~b}$ ). Thus, some second-order spectral points between FOR and the found null may be misidentified as FOPs. Comparing Figure 7a with Figure 7b, we can see that the SSB method misidentifies more second-order points as FOPs than the SeaSonde method. In fact, this may be the common case for FOR overlapping with second-order region. Because, in the tails of the FOR, there are usually some local minima that cannot be smoothed out by the smoothing processing of the SeaSonde method (see Appendix A). Thus, we infer that the SeaSonde method often misidentifies less second-order points as FOPs than the SSB method. In summary, for the edge case of FOR overlapping with second-order region, both the SSB and SeaSonde methods tend to misidentify some second-order spectral points as FOPs, while the SeaSonde method often outputs less error than the SSB method. 


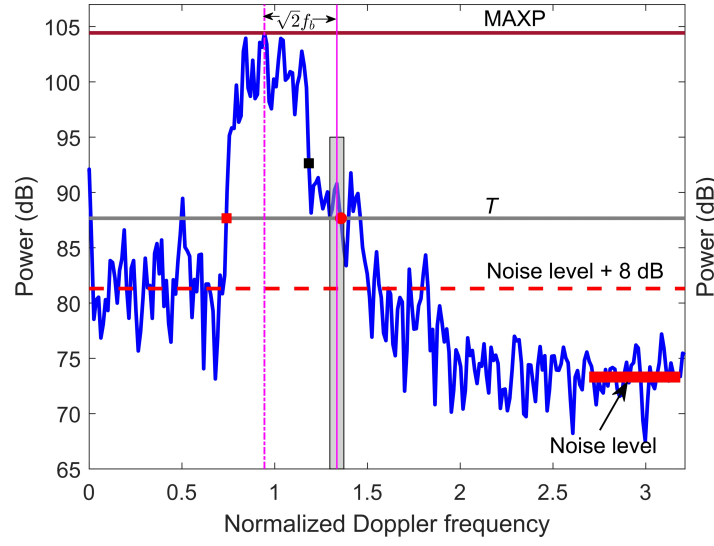

(a)

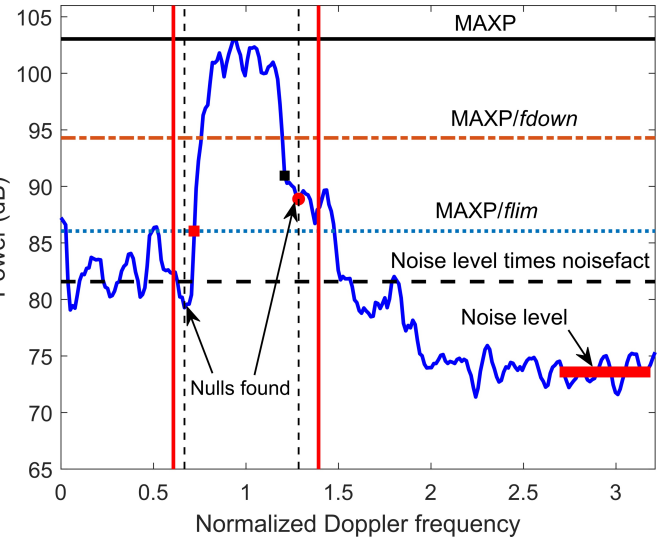

(b)

Figure 7. First-order peaks determination for the case of first-order region overlapping with secondorder region. (a) using the proposed method; (b) using the SeaSonde method. The two red vertical lines in (b) indicate the maximum current limit. The spectral points between the red solid square and the red solid dot are the determined first-order peaks, whereas the spectral points between the red solid square and the black solid square are the visually determined first-order peaks.

\section{Discussion}

The testing results suggest that the SSB method statistically has a similar but not exactly the same performance as the SeaSonde method. The fundamental causes of this performance similarity are as follows: (1) both methods use the spectra feature, which contains nulls between first- and second-order spectra, to separate FOPs in the case of with second-order returns; (2) both methods use a constant SNR to determine FOPs in the case of second-order absent and the settings of the SNR in these two methods are the same value. In addition, the subtle differences of the statistical results between these two methods originate from two aspects. One is that on the tails of the FOR there may be some local minima that can be misidentified as boundaries by the SeaSonde method but have much smaller effects on the SSB method, although there is a prominent null between the first- and second-order region. Another one is that the amplitude threshold calculated from the second-order region in the SSB method is not exactly equal to the threshold used in the SeaSonde method. The performance analyses on the two edge cases suggest that the SeaSonde method is prone to omit some true FOPs, whereas the SSB method tends to produce more FOPs as well as apt to contain more false FOPs than the SeaSonde method. The omission of true FOPs leads to the situation in which some accurate radial current observations are dropped. Containing false FOPs results in spurious radial current observations. At present, a few omissions of the FOPs can be partially compensated by spatial gap-filling techniques [26,27]. Also, a few spurious observations can be handled by spatial smoothing or despiking the radial velocity time series via standard methods [28,29]. Unfortunately, no technique can compensate for a lot of omissions. However, a lot of spurious observations may be handled by advanced quality assurance-quality control (QA-QC) technique (e.g., the non-velocity metric-based QA-QC method in [30]). Nevertheless, more work is needed to understand how the tendency of selecting false FOPs can be addressed in a systematic way. For this reason, and to spur additional development of HFR signal processing in a coherent way, the MATLAB-based code for the SSB method is being made available in https:/ / github.com/260846137/SSB-FOPS.git.

The most remarkable advantage of the SSB method is that it only needs to set one parameter, in contrast to the array of parameters needed in the SeaSonde method. Furthermore, the parameter needed in the SSB method is also needed in the SeaSonde method. Undoubtedly, simplifying input parameters is very attractive to HFR users, especially to those who are not proficient in the theory of current mapping by HFR. Because fewer input parameters mean easier to use and less parameter tuning work. In addition, the simplifica- 
tion of the input parameter for the SSB method does not seem to affect its flexibility, because the statistical results for a such long-term observation suggest that the FOPs determination results from the two methods are still comparable. Moreover, the SSB method seems more robust than the SeaSonde method. This can be inferred from Figure 4 where there is no outlier above the fitted line (or for $N_{p r o}$ ), but there are lots of outlier below the fitted line (or for $N_{\text {cum }}$ ). On the other hand, although the SSB method is tested on a data set collected by OSMAR-S HFR system, this FOPs determination method also can be applied to other HFR systems. The OSMAR-S produces binary files storing the voltage amplitude which can be used to obtain range-Doppler spectra by twice Fourier Transformation. Other HFR systems may collect different variables, but the Doppler spectrum still can be derived. The Doppler spectrum is exact the processing object in the SSB method. Thus, just as the SeaSonde method, the SSB method has good cross-applicability to different HFR systems.

\section{Conclusions}

An alternative methodology for FOPs determination is presented that only needs one user-defined parameter, which is the maximum current velocity that will be encountered by the radar. In comparison, existing FOR determination methods need an array of user-defined parameters. Thus, the proposed method can reduce the number of input parameters and simplify the tuning process on the user-defined parameters. Testing the proposed method on a 256-day field observation data set suggests that the proposed method produces a similar result with the SeaSonde method. Although the performance of the proposed method is only similar to the existing methods and has no prominent improvements, future expansions of this method to other operational HFR systems are likely to simplify the use and popular with users who are unprofessional in the HFR. Thus, an input-parameter-reduced method is significant for promoting the popularization and application of the HFR. Moreover, the performance assessment approach introduced in this study can be extended to assess other first-order peaks determination methods.

Author Contributions: Conceptualization, Y.L.; methodology, Y.L.; software, Y.L.; validation, Y.L.; formal analysis, Y.L.; investigation, Y.L.; resources, H.Z.; data curation, H.Z.; writing—original draft preparation, Y.L.; writing—review and editing, Y.L.; supervision, Y.W.; project administration, Y.W.; funding acquisition, Y.L. and Y.W. All authors have read and agreed to the published version of the manuscript.

Funding: This work was supported by the National Natural Science Foundation of China (NSFC) project (41906171), the China Postdoctoral Science Foundation projects (2019M652286, 2020T130275), and the Postdoctoral Science Foundation of Jiangxi Province (2019KY60).

Institutional Review Board Statement: Not applicable.

Informed Consent Statement: Not applicable.

Data Availability Statement: Restrictions apply to the availability of these data. Data was obtained from Hainan Ruihai Ocean Technology Co. Ltd. and are available from Yeping Lai with the permission of Hainan Ruihai Ocean Technology Co. Ltd.

Conflicts of Interest: The authors declare no conflict of interest.

\section{Appendix A. The SeaSonde Method}

Figure A1 shows a typical HF radar echo spectrum. Apparently, there are local minima (nulls) between the first- and second-order spectra. To determine the FOR, the underlying principle of the SeaSonde method is to find these nulls. Consequently, these nulls are regarded as the FOR boundaries, and the spectral points within these two pairs of the nulls are identified as FOPs. 


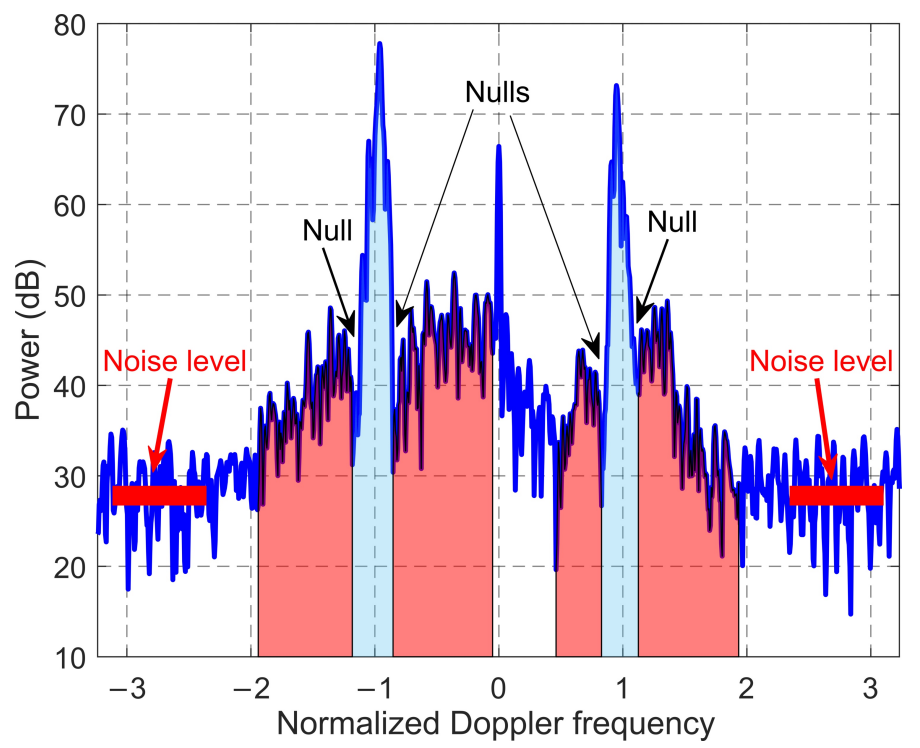

Figure A1. Typical Doppler spectrum for showing the first-order spectra (shaded with light blue) separated by nulls from the second-order spectra (shaded with light red), and the noise level, which is indicated by the thick red lines in the wings of the spectrum and is calculated as the mean power of the spectral points enclosed by the two rectangles.

Now, we describe the detailed steps of the SeaSonde method in determining the FOPs. Figure A2 summarizes the processing flow of the SeaSonde method. For a Doppler spectrum (e.g., Figure A1), the SeaSonde method uses five steps to determine FOPs.

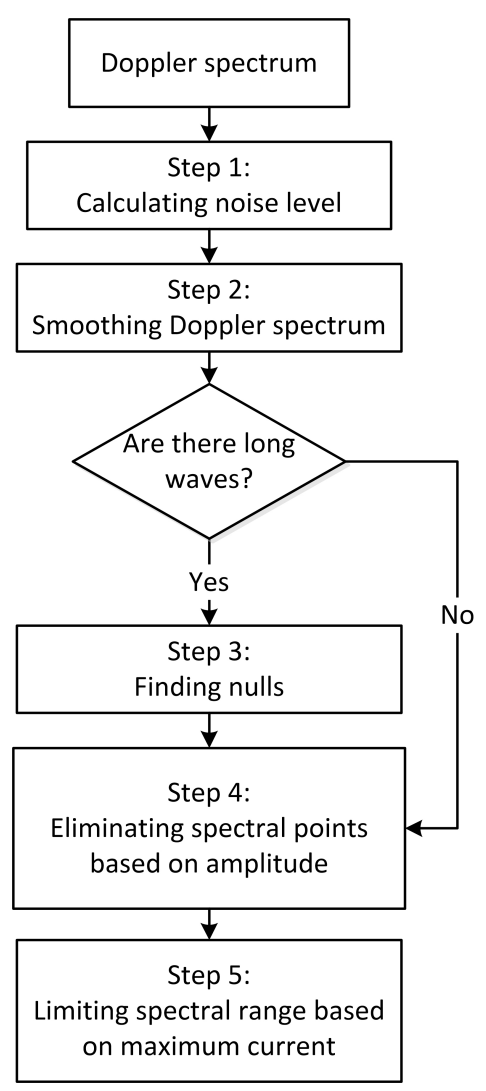

Figure A2. The processing flow of the SeaSonde first-order determination method. 
Step 1: calculating the noise level. Generally, Doppler bins in the wings of the spectrum only contain noise. Thus, the SeaSonde method adopts the averaged power of the Doppler bins in the wings as the noise level for the spectrum, but a specific Doppler region used to calculate the noise level has not been presented in the SeaSonde technical document [14]. In this study, the Doppler bins with normalized frequency within 2.7 to 3.2 and -3.2 to -2.7 are empirically used to calculate noise level. As Figure A1 shows, the two short thick red lines indicate the derived noise level (which is $55.94 \mathrm{~dB}$ ) for the spectrum, and the spectral points enclosed by the two rectangles are the specific spectrum region used to calculate the noise level. This noise level, in step 4, will be used to eliminate Doppler bins that have a power below this noise level times noisefact. (noisefact is a factor defined in SeaSonde technical document [14], and its default value is 4.0.)

Step 2: smoothing the spectrum. To increase the stability in finding the nulls between first- and second-order spectra, a moving average is applied to the spectrum with a window length of $n s m$ Doppler bins. Figure A3 shows the smoothed spectrum of the spectrum displayed in Figure A1 with nsm being equal to 3, 5, and 29. From this figure, we can see that a too large value of $n s m$ (e.g., $n s m=29$ ) may destroy the null between first- and second-order spectra, which may cause errors in setting the boundaries. On the other hand, a too small value of $n s m($ e.g., $n s m=3$ ) may result in local minima within the FOR. These local minima may be identified as a null. Consequently, some true FOPs may be eliminated. Thus, a suitable value should be set for eliminating local minima within FOR and avoiding expected null destruction. Fortunately, $n s m$ often can be set in a large span. In other words, the nulls between first- and second-order regions are insensitive to the value of $n s m$. For the spectrum displayed in Figure A1, the setting of $n s m=5$ effectively eliminates local minima within the FOR and retains the nulls between the first- and secondorder regions (Figure A3). In fact, the expected nulls between the first- and second-order regions remain even for $n s m=21$. Thus, for this spectrum, $n s m$ can be set in a large span from 5 to 21. In this study, we set $n s m=5$ for the SeaSonde method.

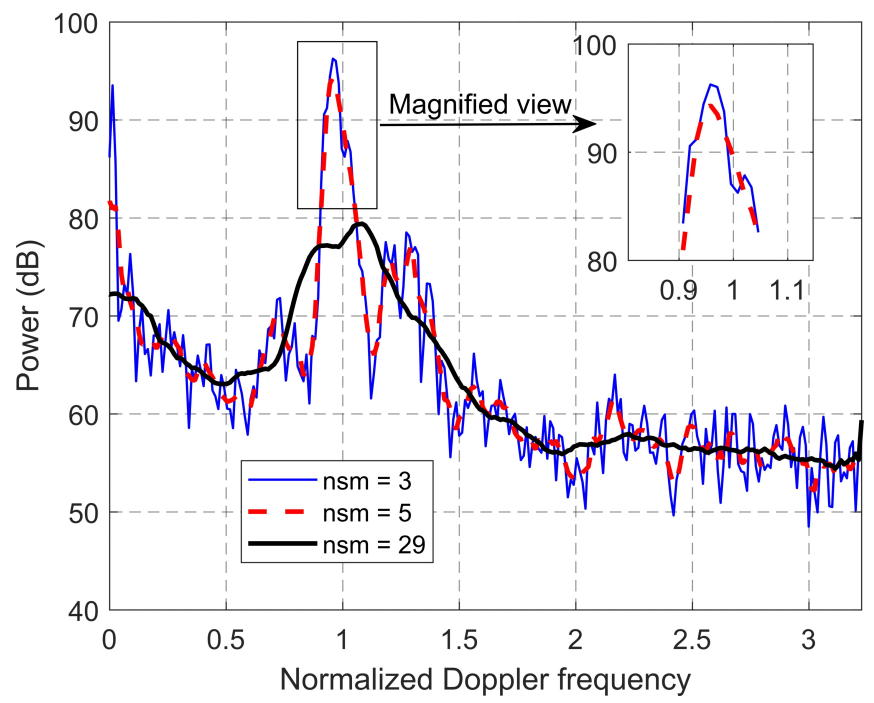

Figure A3. Smoothed spectra of the spectrum displayed in Figure A1. nsm is the window length for moving average. Magnified views for $n s m=3$ and $n s m=5$ in the red box are shown in the top right corner. There is a local minimum (near the normalized frequency of 1 ) within FOR for $n s m=3$. Null between first- and second-order is smeared out for $n s m=29$.

Step 3: finding the nulls between first- and second-order spectra. The first-order regions (FORs) are usually separated from the second-order spectra by conspicuous minima, which we term nulls. When locating these nulls, we should avoid searching for them within the FOR, which may have its own local minima that are not smoothed out. So the search is begun on the periphery of the FOR. More precisely, the search starts at the spectral 
point where the surrounding spectral points have a power of MAXP/fdown, with MAXP being the maximum power and fdown being a factor. These are indicated in Figure A4 for fdown $=2(3 \mathrm{~dB})$ and fdown $=7.5(8.75 \mathrm{~dB})$. From Figure A4 we can see that setting fdown to a too small value will result in that local minima within FOR is misidentified as nulls, meaning that some true FOPs are eliminated. Certainly, if fdown is chosen too large, the nulls may be missed altogether, resulting in second-order spectrum points included and resulting in wild current vectors. For the spectrum in Figure A4, fdown $=7.5$ results in acceptable nulls. In fact, the default value of this parameter, fdown, is 7.5 in the SeaSonde system. In this study, we also use this default value for fdown. On the other hand, care must be taken for this step. If there are no long waves on the water surface, as in a harbor, there will be no second-order sea echoes. In this case, this step should be skipped by setting the parameter nsec to zero.

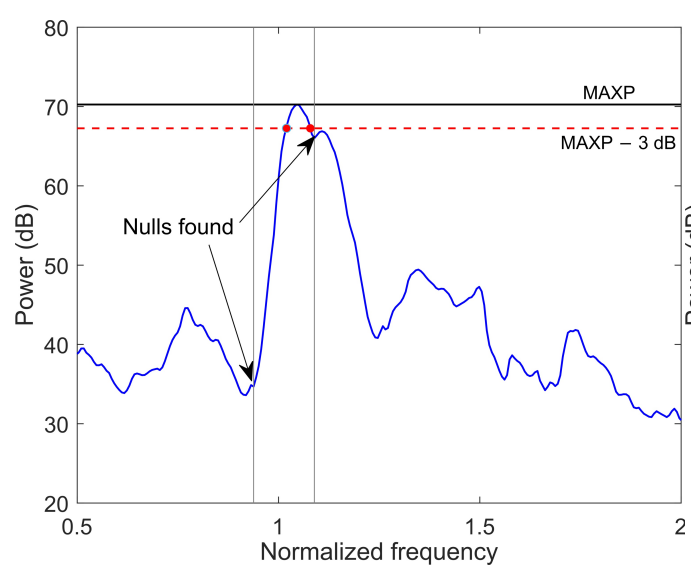

(a)

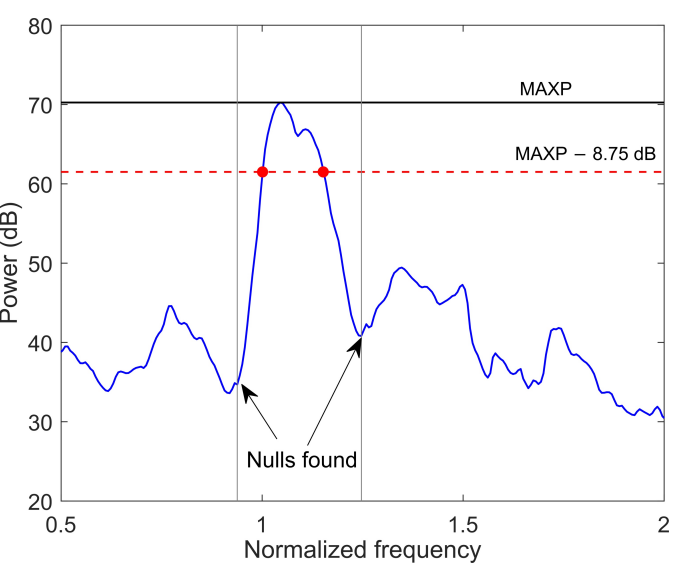

(b)

Figure A4. Demonstrates the effect of different values of the parameter fdown on finding the nulls between first- and second-order region. (a) unsuccessfully; (b) successfully. The red dots indicate the positions at which the search for the nulls starts.

Step 4: eliminating spectral points based on amplitude or power. The spectral points within a pair of nulls (e.g., Figure A4b) may include some bad points, which are often adjacent to the nulls. Thus, the SeaSonde method uses two parameters, flim and noisefact, to eliminate spectral points, which are too far below the peak energy or have a low SNR. Specifically, spectral points with power being less than MAXP divided by flim or less than noisefact times the noise level (calculated in step 1) are eliminated. The default values for flim and noisefact are $15(11.76 \mathrm{~dB})$ and $4(6.02 \mathrm{~dB})$, respectively. In our study, we set the values of flim and noisefact to $50(16.99 \mathrm{~dB})$ and $6.3(8.00 \mathrm{~dB})$, respectively.

Step 5: Limiting spectral range based on maximum current velocity. In the final step, the SeaSonde method applies a frequency window determined by the maximum current (currmax), which is estimated for the geographical location of the radar. Thus, the derived current velocity will not exceed this predefined maximum current. In this study, this maximum current velocity is set as $1.5 \mathrm{~m} / \mathrm{s}$. Apparently, the order of step 5 and step 4 can be exchanged without any effect on the result of the determined FOPs. In addition, steps 3, 4, and 5 are applied separately for the positive and negative Doppler halves of the Doppler spectrum.

\section{References}

1. Paduan, J.D.; Washburn, L. High-Frequency Radar Observations of Ocean Surface Currents. Annu. Rev. Mar. Sci. 2013,5 , 115-136. [CrossRef] [PubMed]

2. Mantovani, C.; Corgnati, L.; Horstmann, J.; Rubio, A.; Reyes, E.; Quentin, C.; Cosoli, S.; Asensio, J.; Mader, J.; Griffa, A. Best Practices on High Frequency Radar Deployment and Operation for Ocean Current Measurement. Front. Mar. Sci. 2020, 7, 210. [CrossRef] 
3. Jena, B.; Arunraj, K.; Suseentharan, V.; Tushar, K.; Karthikeyan, T. Indian Coastal Ocean Radar Network. Curr. Sci. 2019, 116, 372-378. [CrossRef]

4. Roarty, H.; Cook, T.; Hazard, L.; George, D.; Harlan, J.; Cosoli, S.; Wyatt, L.; Alvarez Fanjul, E.; Terrill, E.; Otero, M.; et al. The Global High Frequency Radar Network. Front. Mar. Sci. 2019, 6, 164. [CrossRef]

5. Barrick, D. First-Order Theory and Analysis of MF/HF/VHF Scatter from the Sea. IEEE Trans. Antennas Propag. 1972, 20, 2-10. [CrossRef]

6. Stewart, R.H.; Joy, J.W. HF Radio Measurements of Surface Currents. Deep. Sea Res. Oceanogr. Abstr. 1974, 21, 1039-1049. [CrossRef]

7. Barrick, D.; Lipa, B.; Crissman, R. Mapping Surface Currents with CODAR. Sea Technol. 1985, 26, 43-48.

8. Graber, H.C.; Haus, B.K.; Chapman, R.D.; Shay, L.K. HF Radar Comparisons with Moored Estimates of Current Speed and Direction: Expected Differences and Implications. J. Geophys. Res. Ocean. 1997, 102, 18749-18766. [CrossRef]

9. Gurgel, K.W.; Antonischki, G.; Essen, H.H.; Schlick, T. Wellen Radar (WERA): A New Ground-wave HF Radar for Ocean Remote Sensing. Coast. Eng. 1999, 37, 219-234. [CrossRef]

10. Barrick, D.E.; Evans, M.W.; Weber, B.L. Ocean Surface Currents Mapped by Radar. Science 1977, 198, $138-144$.

11. Lipa, B.; Barrick, D. Least-Squares Methods for the Extraction of Surface Currents from CODAR Crossed-Loop Aatenna: Application at ARSLOE. IEEE J. Ocean. Eng. 1983, 8, 226-253. [CrossRef]

12. Liu, Y.; Weisberg, R.H.; Merz, C.R. Assessment of CODAR SeaSonde and WERA HF Radars in Mapping Surface Currents on the West Florida Shelf. J. Atmos. Ocean. Technol. 2014, 31, 1363-1382. [CrossRef]

13. Emery, B.; Washburn, L. Uncertainty Estimates for SeaSonde HF Radar Ocean Current Observations. J. Atmos. Ocean. Technol. 2019, 36, 231-247. [CrossRef]

14. COS. Defining First-Order Region Boundaries. CODAR Ocean Systems Technical Manual. 2002. Available online: http: //support.codar.com/Technicians_Information_Page_for_SeaSondes/Docs/Informative/FirstOrder_Settings.pdf (accessed on 22 December 2020).

15. COS. SeaSonde Radial Suite Release 7; CODAR Ocean Sensors (COS): Mountain View, CA, USA, 2013.

16. Kirincich, A. Improved Detection of the First-Order Region for Direction-Finding HF Radars Using Image Processing Techniques. J. Atmos. Ocean. Technol. 2017, 34, 1679-1691, [CrossRef]

17. Crombie, D.D. Doppler Spectrum of Sea Echo at 13.56 Mc./s. Nature 1955, 175, 681-682. [CrossRef]

18. Ivonin, D.V.; Shrira, V.I.; Broche, P. On the Singular Nature of the Second-Order Peaks in HF Radar Sea Echo. IEEE J. Ocean. Eng. 2006, 31, 751-767. [CrossRef]

19. Zhou, H.; Wen, B. Wave Height Estimation Using the Singular Peaks in the Sea Echoes of High Frequency Radar. Acta Oceanol. Sin. 2018, 37, 108-114. [CrossRef]

20. Tian, Y.; Wen, B.; Tan, J.; Li, Z. Study on Pattern Distortion and DOA Estimation Performance of Crossed-Loop/Monopole Antenna in HF Radar. IEEE Trans. Antennas Propag. 2017, 65, 6095-6106. [CrossRef]

21. Ivonin, D.V.; Broche, P.; Devenon, J.L.; Shrira, V.I. Validation of HF Radar Probing of the Vertical Shear of Surface Currents by Acoustic Doppler Current Profiler Measurements. J. Geophys. Res. Ocean. 2004, 109, [CrossRef]

22. Tian, Z.; Tian, Y.; Wen, B.; Wang, S.; Zhao, J.; Huang, W.; Gill, E.W. Wave-Height Mapping From Second-Order Harmonic Peaks of Wide-Beam HF Radar Backscatter Spectra. IEEE Trans. Geosci. Remote. Sens. 2020, 58, 925-937. [CrossRef]

23. Lai, Y.; Zhou, H.; Zeng, Y.; Wen, B. Accuracy Assessment of Surface Current Velocities Observed by OSMAR-S High-Frequency Radar System. IEEE J. Ocean. Eng. 2018, 43, 1068-1074. [CrossRef]

24. Lai, Y.; Zhou, H.; Zeng, Y.; Wen, B. Quantifying and Reducing the DOA Estimation Error Resulting from Antenna Pattern Deviation for Direction-Finding HF Radar. Remote Sens. 2017, 9, 1285. [CrossRef]

25. Lai, Y.; Zhou, H.; Zeng, Y.; Wen, B. Relationship Between DOA Estimation Error and Antenna Pattern Distortion in DirectionFinding High-Frequency Radar. IEEE Geosci. Remote Sens. Lett. 2019, 16, 1235-1239. [CrossRef]

26. Saha, D.; Deo, M.C.; Bhargava, K. Interpolation of the Gaps in Current Maps Generated by High-Frequency Radar. Int. J. Remote Sens. 2016, 37, 5135-5154. [CrossRef]

27. Fredj, E.; Roarty, H.; Kohut, J.; Smith, M.; Glenn, S. Gap Filling of the Coastal Ocean Surface Currents from HFR Data: Application to the Mid-Atlantic Bight HFR Network. J. Atmos. Ocean. Technol. 2016, 33, 1097-1111. [CrossRef]

28. Halle, C. HF Radar Processing Using "Nearest-Neighbor" Statistics; Techreport; University of California, Davis, Bodega Marine Laboratory: Davis, CA, USA, 2008; 26p.

29. NDBC. Handbook of Automated Data Quality Control Checksand Procedures; NOAA/NDBC: Hancock County, MS, USA, 2009; 78p.

30. Kirincich, A.R.; De Paolo, T.; Terrill, E. Improving HF Radar Estimates of Surface Currents Using Signal Quality Metrics, with Application to the MVCO High-Resolution Radar System. J. Atmos. Ocean. Technol. 2011, 29, 1377-1390. [CrossRef] 